TITLE:

\title{
$<$ Forum> Establishing a long-term veterinary project for free-ranging chimpanzees in Tanzania
}

AUTHOR(S):

Lukasik, Magdalena

CITATION:

Lukasik, Magdalena. <Forum> Establishing a long-term veterinary project for free-ranging chimpanzees in Tanzania. Pan Africa News 2002, 9(2): 13-17

ISSUE DATE:

2002-12

URL:

http://hdl.handle.net/2433/143417

RIGHT:

Copyright (C) Pan Africa News. 
<FORUM>

Establishing a Long-term Veterinary Project for Free-Ranging Chimpanzees in Tanzania

\author{
Magdalena Lukasik, MDV \\ Jane Goodall Institute, Gombe Stream \\ Research Center, P.O. Box 185 Kigoma
}

Generally speaking, Pan troglodytes is not an endangered species. Not as the whole species. There are still many-probably too many, chimpanzees in captivity. But situation is very different when it comes to the free-ranging communities.

The range of problems jeopardising their existence is wide: bush-meat trade in Central and Western Africa, deforestation all around their natural habitat, finally: antropozoonotic diseases, which so easily cross the inter-species barrier between human researchers, tourists and local inhabitants to the apes in forest.

There are no records of chimpanzee hunting 
in Tanzania, but two latter factors combined caused the death of about $25 \%$ population of chimpanzees in the Gombe National Park in Tanzania in the last decade. Reports from the Mahale Mountains National Park also indicate recent noticeable decrease in the ape population.

Due to scale of the problem and variety of factors involved, it seems inevitable that people involved in various fields of research, conservation and education join their forces to stop this process. Because diseases, especially the anthropozoonoses, are responsible for the death of many of these animals (in Gombe at least 16 chimpanzees died due to antropozoonotic illnesses in only last 6 years), it seems important that the team includes also the veterinary component.

There are wildlife veterinarians working in Tanzania, who can be invited to collaboration, but in case of great apes some specific knowledge and skills are required comparing to working with other African wildlife.

There are three facts to explain such an opinion. First, unlike most other wildlife veterinarians, an ape doctor works on foot, following his patients around thick forest, carrying all equipment, for many hours in a row. Second, dealing with the animals of such complex behaviour and social life, it is very important to spend lot of time learning to understand their reactions to different situations, including the health disorders. In order to gather this knowledge veterinarian has to spend reasonable time just following his targets and watching them carefully for many hours. Also, especially when darting wild chimpanzees is very difficult, noninvasive sampling is a main part of practice. Again, this requires long-term, regular follows.

Third reason is due to genetical closeness of apes and humans, which means that doctor and patient can possibly share almost all pathogens, which therefore creates considerable risk for both of them. Also, because apes are closer related to people than other animals, working with them often requires close collaboration with human doctors and laboratories rather than these of veterinary medicine.

The possible solution for the problem is to establish the project specialised in working with wild chimpanzees, following the example of Morris Animal Foundation Mountain Gorilla
Veterinary Project working in Virunga National Park in Rwanda, Uganda and Congo-Zaire.

The attempts to establish similar Project for free-ranging chimpanzees have started in Tanzania in October 2001, when the Chimpanzee Health Project has been established within Gombe Stream Research Center JGI in Gombe National Park. After one year of this study, which was the pilot one, conducted without any veterinary facility yet present, lots of valuable information about such project requirements and possibilities were gathered.

The Project work consisted of four main parts:

1. Everyday monitoring of the chimpanzee health status, including observation and sample collection from both healthy and sick animals.

2. Autopsy of every animal-including other species, found dead in the forest.

3. Investigation and, in certain cases, treatment during general disease outbreaks.

4. Close collaboration with TANAPA and Researchers in order to decrease the risk of antropozoonotic and zoonotic diseases within the Park.

It included the regular social work programs: teaching (health seminars), vaccination in case of disease risk and general investigation to estimate the health status of the human and non-human primates living in Gombe National Park.

\section{Monitoring: Observation and Non- Invasive Sample Collection}

As indicated by the pilot study, if there is an ongoing research project at the site, this part of work can and preferably should be done in collaboration with researchers and their assistants.

The credits of such collaboration are substantial and mutual.

Due to the risk of antropozoonotic diseases it is highly advisable to decrease number of people in contact with chimpanzees. Involving researchers in basic veterinary monitoring and sampling seems the best policy allowing to obtain more thorough data without presence of additional veterinary assistants in forest.

Also, because of their everyday presence around animals and good knowledge of chim- 
panzee behaviour, researchers of diet and range are able to notice many abnormalities sooner than veterinarian, who lacks this experience. So not only they can be help with the data and sample collection, but also may serve as the teachers for the veterinary team.

If the simple Health Monitoring Sheets are used, the basic information can be recorded on every day base and the data compared within the long period of time. These sheets should be easy to understand by the non-vet assistants and ideally always carried by them during the forest work.

On the other hand, involving veterinarian in behavioural research often helps explain certain abnormalities of behaviour connected with a disease.

Also post mortem findings can help understand some unusual behaviour and a vet, following the pattern of injuries and autopsy finding can help understand some facts of predatory and intra- and intercommunity aggression. In addition a material collected for veterinary purpose can be simultaneously used for other research purposes, for example urine collection for regular pregnancy testing or fecal sampling for the paternity study.

Apart from the data collected during observation, in order to obtain the clear picture of community health status, regular, everyday sample collection should also be conducted.

Non-invasive sampling is, due to the risk and difficulty involved in darting of free-raging chimpanzees, the method of choice for gathering the biological material from this species. Unlike the sampling involving anaesthesia, it provides the regular, even everyday source of material.

Due to the rapid evolution of laboratory techniques it is also the potential basis for wide variety of different tests.

\section{MATERIAL AVAILABLE FOR NON-INVASIVE} COLLECTION FROM FREE-RANGING CHIMPANZEES:

- Feces (bacteria, virus, parasites, cytology, hormones, DNA)

- Urine (bacteria, virus, antibodies, chemistry, parasites, cytology, hormones)

- Wadge (bacteria, virus, cytology, DNA)

- Semen (bacteria, virus, DNA)
- Hair (parasites, DNA)

- Discharges: pus, sputum, exudates (bacteria, virus, parasites, cytology, DNA)

- Blood-very small amount obtainable (bacteria, virus, parasites, antibodies, DNA).

\section{Autopsy}

Simultaneously to being the important source of diagnostic material, autopsy work with chimpanzees creates, much bigger than in case of other species, risk for the humans involved. It is extremely important to provide the safe conditions for this work, including the separate post mortem room and limiting number of people who have the contact with potentially highly infectious material.

Because of many pathogens shared by different primates, and even non-primate species in forest, it is important that any animal found dead in a forest should be carefully examined and samples collected for the future laboratory testing.

\section{Disease Investigation and Treatment}

As in case of everyday health monitoring, investigation during disease outbreaks should also be based on non-invasive sampling. Darting should be restricted to the cases when it is absolutely necessary and no other method of work can be used instead. Apart from the risks involved and the fact that a free ranging chimpanzee would probably let himself to be darted only once in a lifetime, in some cases it has been proved that the action had led to result no different than what would anyway happen in natural way. Therefore it should always be individually considered if the risk involved in the action does not create the bigger risk than the injury itself. In case it is undertaken, the carefully calculated combination of the oral and injectable drugs should be used to minimize the danger. There are, of course, situations when anesthesia is the method of choice: for example when animal is found still kept by the snare or darting can be undertaken before the necrosis of tissue appeared.

In case when disease makes an animal very weak or unconscious, it is often possible to dart, or inject directly with the therapeutic drug (anti- 
biotic, steroid, etc) without administration of anaesthetic.

Unlike in the work with gorillas, in veterinary of free-ranging chimpanzees bases mainly on the oral medicine administration, which is easier, less risky and usually not less effective.

Still this method can be used only in habituated animals and in case when they are still strong enough to be interested in food bites.

While the single case of disease in habituated chimpanzee is considerably easy to treat, it becomes much more difficult in a situation of general outbreak. It is extremely hard to monitor and administrate medicine to a big number of free-ranging animals and there is a big risk of loosing many-sometimes up to $50 \%$ of $\mathrm{com}^{-}$ munity members. Therefore it is essential to prevent such outbreaks through the policy of rules for all the people in contact with chimpanzees.

This policy includes the vaccination programs, hygiene control and education and the rules for researchers, tourists and all the people living in the forest.

\section{List of Diseases Considered as Most Jeopardising for the Free-Ranging Apes}

\section{BACTERIAL INFECTIONS:}

Shigella, Salmonella, Campylobacter, Mycobacterium (tuberculosis and bovis, possibly also avium), Streptococcus pneumoniae, very probably also Neisseria meningiditis and Haemophilus influenzae.

\section{VIRAL INFECTIONS:}

Herpes simplex, Chicken pox, Measles, Mumps, Polyo, Rota, Hepatitis A and B, Influenza viruses, Parainfluenza type 3, Resp. Syncytial virus.

\section{PARASITE INFECTIONS:}

Entamoeba histolytica, Giardia lamblia, Balantidium coli, Sarcoptes scabiei.

(Ott-Joslin, Zoo and wild animal medicine, Jones et al. 1984, Speare 97, Brack 87).

Of which the humans working and living within the chimp habitat should be vaccinated or tested for: Hepatitis A and B, Yellow fever,
Meningococcus, Polyo, Measles, Mumps, Rubella, and actual (at most one year old) tuberculosis test.

Apart from vaccination programs, there are certain attributes of forest work, which can be changed in order to minimize the risk of antropozoonotic infection.

As the forest animals frequently visit camp, it is extremely important that the area is clean, houses well-protected and number of human inhabitants decreased only to the necessary minimum. All residents of Park should be regularly examined in the local hospital. Examination should include the fecal and blood test in each case and additional diagnostic method suggested during the general clinical check up. Also the general vaccination program should be carefully considered.

It is also essential that during their work in forest researchers and tourist guides follow the list of rules describing quarantine, distances and time spent with animals. Only the good will and close collaboration between TANAPA and researchers can make the situation better.

\section{Social work.}

During the pilot study the veterinary project in Gombe has also been involved in wide variety of social work, including:

- Open health seminars for research assistants and TANAPA employees working and living inside the park. These seminars were prepared and presented together with TANAPA nurse and covered the subjects of general biology, anatomy and hygiene.

- Collaboration with TANAPA in order to create the list of animal safety rules for people working and leaving in Gombe, as well as visiting researchers and tourists. Since such list is ready, the work is concentrated on practical aspect of rules being followed.

- Collaboration in work of GOMBE HEALTH COMMITEE, formed together with TANAPA directory of the Park.

It proved very beneficial for the general understanding of health issues among the people living and working in Park and seems a highly advisable component for the Chimpanzee Veterinary Project work. 


\section{Conclusions After 14 Months of Project Work}

1. Chimpanzee Veterinary Project in Tanzania should be extended to cover also other areas where these animals live. There is about 1000 chimps in the country: Gombe-100, Mahale700, Rubondo-40, plus chimps still living in non-protected areas, and about $90 \%$ of them are not included in the Veterinary Program.

2. The location of Project should be carefully considered. For the everyday work Project requires small veterinary facility, including post mortem room and basic laboratory. Because Gombe is the smallest (about $40 \mathrm{~km}^{2}$ ) and most crowded (about 150 people living within the Park boundaries), it does not seem the good idea to bring any more people or building there. As most of Tanzanian chimpanzees live in the Mahale Mountains NP, which is also the biggest chimpanzee habitat in the country, with the good communication (Lake Tanganyika) with Gombe, it seems to be much better place for location of Vet. Project for chimpanzees in Tanzania.

3. Collaboration. If possible, few other organisations, such as TANAPA, TAWIRI and GRASP should collaborate closely within the Project, including also a long-term co-operation with other Ape Veterinary Projects abroad, as Mountain Gorilla Veterinary Project in Rwanda and Uganda and vets working with wild and sanctuary chimps in other countries. Working together would be a great help during emergency, like general disease outbreak. Also unifying policy of work and data sheets used in different sites would allow data to be more general and comparable between the sites.

4. There should be two veterinarians, including at least one Tanzanian, to carry on the work and it also seems important for Project to be involved in wildlife veterinary education in the country. 